\title{
A Gesture-Driven Multimodal Interactive Dance System
}

\author{
Gang Qian ${ }^{\dagger \ddagger}$, Feng Guo ${ }^{\ddagger}$, Todd Ingalls ${ }^{\dagger}$, Loren Olson ${ }^{\dagger}$, Jody James ${ }^{\dagger}$ and Thanassis Rikakis ${ }^{\dagger}$ \\ ${ }^{\dagger}$ Arts, Media and Engineering Program and \\ ${ }^{\ddagger}$ Department of Electrical Engineering \\ Arizona State University \\ Tempe, AZ 85287-8709
}

\begin{abstract}
In this paper, we report a real-time gesture driven interactive system with multimodal feedback for performing arts, especially dance. The system consists of two major parts: a gesture recognition engine and a multimodal feedback engine. The gesture recognition engine provides real-time recognition of the performer's gesture based on the 3D marker coordinates from a marker-based motion capture system. According to the recognition results, the multimodal feedback engine produces associated visual and audio feedback to the performer. This interactive system is simple to implement and robust to errors in 3D marker data. Satisfactory interactive dance performances have been successfully created and presented using the reported system.
\end{abstract}

\section{Introduction}

Recently, interactive performance has attracted great interests in the performing arts. It produces new approaches to the creation and presentation of performing art works. For example, in traditional dance performance, the dancers need to follow the background music with pre-choreographed steps and movements. Although improvisation is possible, the background music will not change automatically according to the dancers movements. In an interactive environment such as the one presented in this paper, dancers become the true masters of the stage. They have full control of the background music and visual display through their movements.

Different cues related to human moment can be used to drive interactive systems. For example, Camerri et al. [1] have used the Laban movement qualities [2] to guide the background music synthesis. Woo [3] extracted limb movement in terms of speed and trajectory in nine cubes centered at human body. In our approach, we have used a number of static human body gestures (poses) to drive the interactive system. During the system development, we have tackled the real-time marker data cleaning problem and designed proper audio and visual immersive feedback. The complete interactive system is illustrated by Figure 1. This system has been successfully demonstrated during the second annual meeting of the National Arts Technology Network (NATN) in November 2003.

\section{Gesture Recognition Engine}

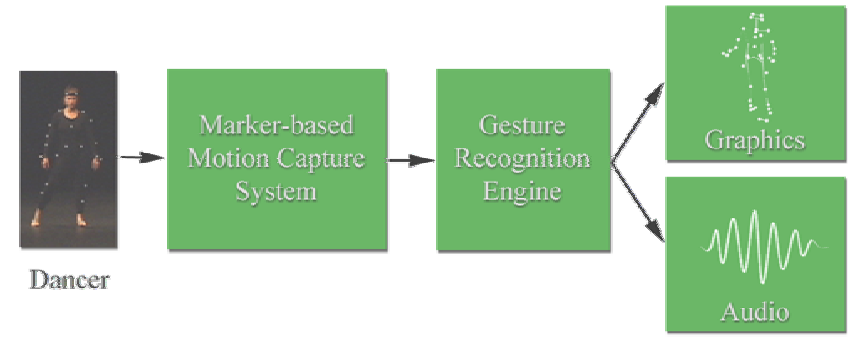

Figure 1. A multimodal interactive dance system
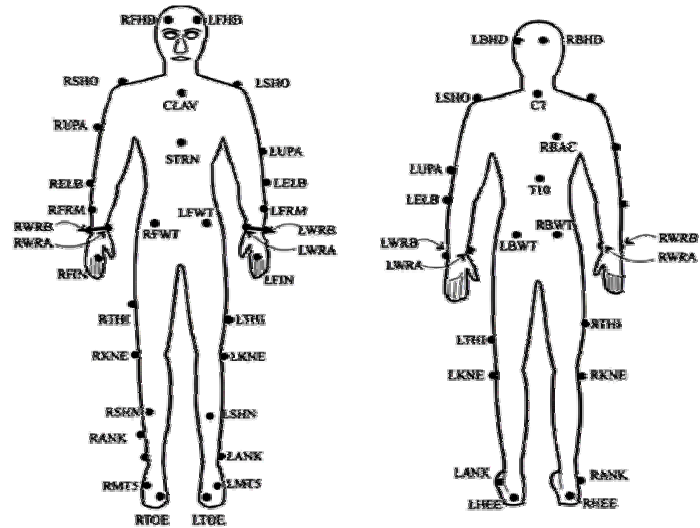

Figure 2. Marker positions (excerpted from VICON user's manual)

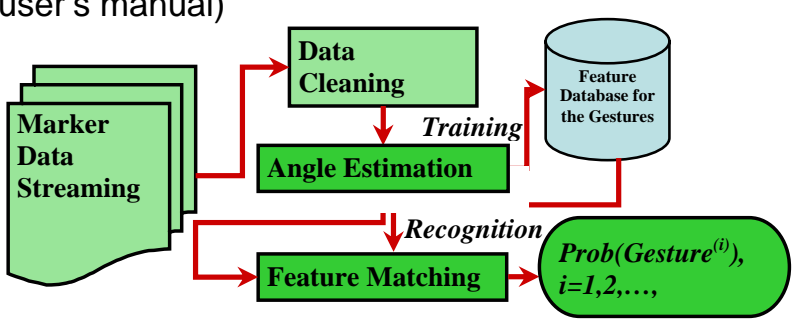

Figure 3. The gesture recognition engine

Marker-based motion capture systems are used to provide real-time marker positions in global space. The gesture recognition engine uses the $3 \mathrm{D}$ marker positions to learn and recognize different gestures. A total of 41 markers are put on the dancer and their positions are shown in Figure 2. The overview of the gesture recognition engine is given by Figure 3 .

\subsection{Feature Extraction}

The torso orientation and joint angles between adjacent body parts and are extracted as features to represent different gestures. The whole human body is 
segmented into 10 body parts, including head, torso, upper arms, forearms, upper legs and lower legs. Feet and hands are ignored in gesture recognition. Each of these 10 body parts is regarded approximately as a rigid object. The local coordinate system with respect to each body part can be constructed and the joint angles between adjacent body parts can be computed.

Table 1. DOF of Joints and Torso

\begin{tabular}{|c|c|c|c|c|c|c|}
\hline Joint & Neck & Shoulder & Elbow & Hip & Knee & Torso \\
\hline DOF & 3 & 3 & 1 & 2 & 1 & 2 \\
\hline
\end{tabular}

Table 1 gives the degrees of freedom of the joints. Regarding torso orientation, the rotation about the body's principle axis is ignored since the gestures with only different facing direction are considered the same. Given head and torso markers, it is straightforward to estimate the rotation angles between the two related local coordinate systems. The joints between upper arms and torso have three DOF. However, we have found that the estimation of the three joint angles is sensitive to marker position noise. To tackle this problem, we use four angles to represent this three DOF joint. Consider shoulder joints. First, the upper arm direction in torso local system is used. This direction will be enough to describe the joint since the rotation about upper arm is not critical when the arm is not folded. When the arm is folded to form a plane, the normal direction of this plane in torso system is also used to represent the shoulder joint. The use of an additional angle increases the stability of the estimation. Hips joint are three DOF joints, but we ignored the rotation about upper leg and only considered hip joints as two DOF joints. For lower limbs one angle is estimated to describe their poses. For example of the elbow, the angle between upper arm and forearm is computed. Hence $X=\left\{X_{1}\right.$, $\left.X_{2}, \ldots, X_{10}\right\}$ is constructed as the feature vector to represent each pose, and $X_{i}$ 's are joint angles vectors with different dimensions of 2, 3 or 4, depending on the particular joint.

\subsection{A Real-time Data Cleaning Algorithm}

Due to the 3D nature of human body, marker occlusion happens during motion capture. To tackle the missing marker problem, we have developed a real-time marker cleaning algorithm. Raw marker data from the motion capture system are cleaned before being fed in angle estimation. As illustrated in Figure 4, two approaches are used to fix a missing marker: static-body-part (SBP) and rigid-body $(R B)$. $S B P$ explores the temporal correlation of the marker position in a pause. If the body part related to a missing marker is static, the previous marker position is used as the current position of the missing marker. The related body part is static when other marker movements on the same body part within recent frames are below a pre-chosen threshold. When the related body part is in motion, $R B$ is used fix the missing marker. $R B$ estimates the $3 \mathrm{D}$ translation and rotation of the body part between the current frame and the model frame, which is obtained

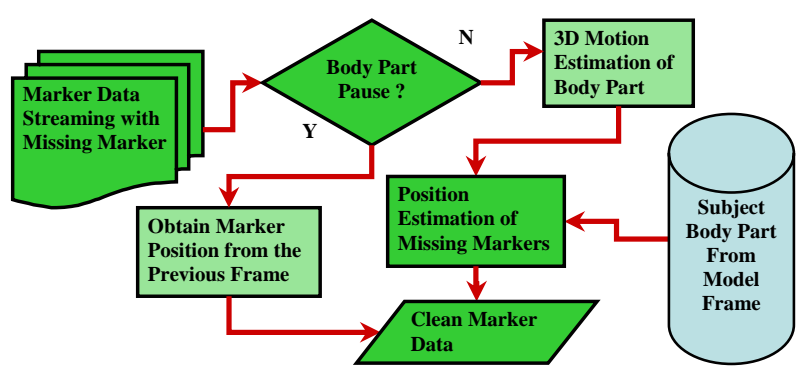

Figure 4. The data cleaning algorithm

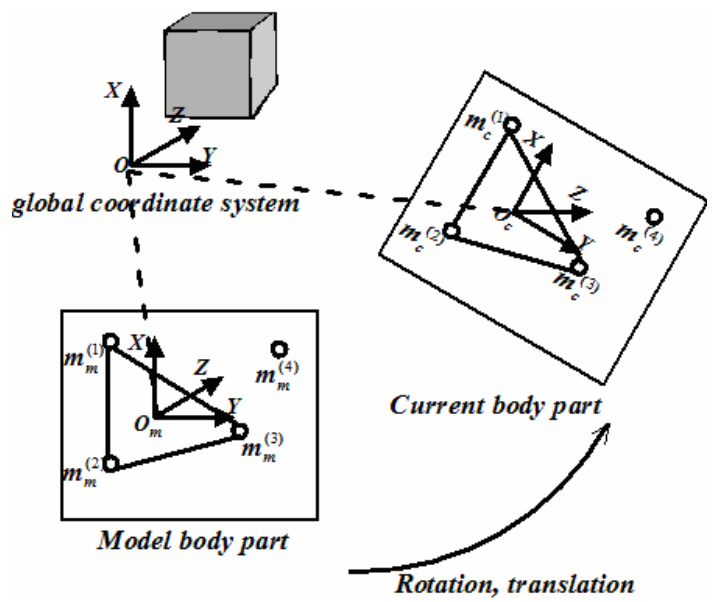

Figure 5. 3D transformation of a body part
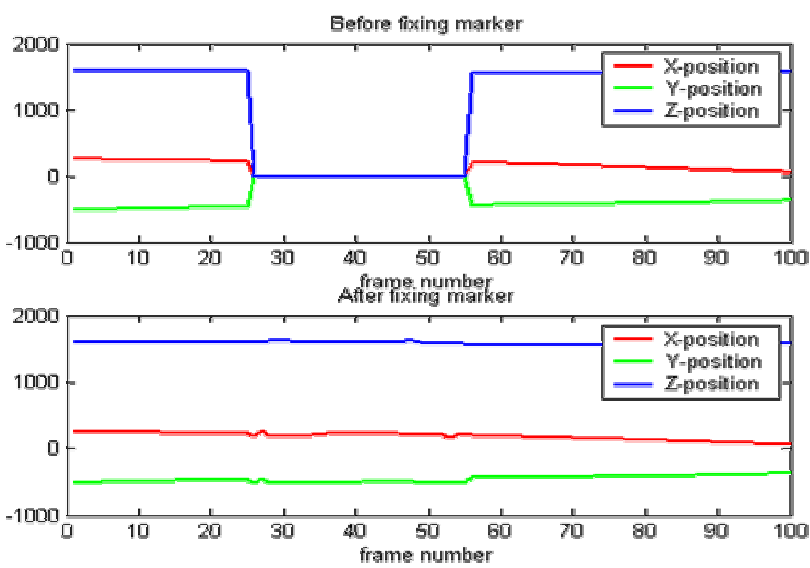

Figure 6. $X-Y-Z$ marker positions before (upper plot) and after (lower plot) data cleaning

from the subject calibration database of the motion capture system. The model frame has the global positions of all markers on each body part. For example, assume that the body parts related to the missing marker has at least 4 markers. Consider Figure 5. Assume that $m_{c}^{(4)}$ is the missing marker in the current frame. Let $m_{c}{ }^{(1)}, m_{c}{ }^{(2)}$ and $m_{c}{ }^{(3)}$ be three non-collinear markers and $O_{c}$ be their centroid in the current frame. Let $m_{m}{ }^{(1)}, m_{m}{ }^{(2)}, m_{m}{ }^{(3)}$ and $O_{m}$ be the positions of the same set of marker and there centorid in the model frame. Because of the rigidity of the 
body part, $m_{c}^{(i)}-O_{c}=R\left(m_{m}^{(i)}-O_{m}\right)$, where $R$ is the rotation matrix between the model and current frame and it can be computed using the visible markers. The coordinate of the missing marker in current frame can be computed by $m_{c}^{(4)}=R\left(m_{m}^{(4)}-O_{m}\right)+O_{c}$. Figure 6 shows the data cleaning results for a missing marker for a hundred frames using the second approach. The big gap in the upper figure was caused by marker occlusion and it was fixed in the lower image.

\subsection{Training}

The same gesture might be executed slightly differently by different people or the same person during different trials. To capture this execution variability, a multivariate Gaussian random vector is used to model the statistical distributions of the joint angles for each gesture, which is given by

$$
p\left(X \mid C_{i}\right)=\prod_{k=1}^{10} \frac{1}{\left|\sum_{i}^{(k)}\right|^{\frac{1}{2}}(2 \pi)^{\frac{d_{k}}{2}}} e^{-\frac{1}{2}\left(X_{k}-\mu_{i}^{(k)}\right)^{T} \Sigma_{i}^{(k)-1}\left(X_{k}-\mu_{i}^{(k)}\right)}
$$

where $C_{i}$ is the $i$ th gesture and $i=1,2, \ldots, G$ is the gesture index. $G$ is the total number of gestures and $d_{k}$ is the dimensional of feature vector $X_{k} \cdot k=1,2, \ldots, 10$ is the body part index. We assume that the joint angle distributions of different body parts are independent. In training procedure, $\left(\mu_{i}^{(k)}, \Sigma_{i}^{(k)}\right)$ related to each body part of all the gestures can be computed.

\subsection{Gesture Matching}

The gesture matching is cast as a multiple hypothesis testing problem. Given the joint angle feature vector of an unknown pose, there are two steps to decide whether this pose is one of the gestures: 1) determine whether this pose is inside the gesture space 2) if it is, the likelihoods of the feature vector given different gestures are computed using the mean and variance variables from the training process. To accomplish the first step, a body part distance matrix $\boldsymbol{D}$ is constructed. $\boldsymbol{D}$ is a $G$-by-10 matrix. (Remember $G$ is the number of gesture and 10 the number of considered body parts). Its component $D_{i, k}$ is the Mahalanobis distance computed using the related parameters by

$$
D_{i, k}=\left(X_{k}-\mu_{i}^{(k)}\right)^{T} \Sigma_{i}^{(k)-1}\left(X_{k}-\mu_{i}^{(k)}\right)
$$

Then, the validity matrix $\boldsymbol{V}$ is computed using $\boldsymbol{D}$ and

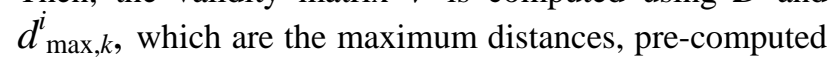
using the training data. $V_{i, k}$ is computed by

$$
V_{i, k}= \begin{cases}1 & D_{i, k} \leq d_{\text {max }, k}^{i} \\ 0 & D_{i, k}>d_{\text {max }, k}^{i}\end{cases}
$$

$I_{i}=\sum^{10}{ }_{k=1} w_{k} V_{i, k}$ indicates the validity of this unknown pose: if any of the $I_{i}$ 's is larger than a threshold, this pose is considered inside the gesture space, where $w_{k}{ }^{\prime} s$ are the weights to emphasize body parts differently: (head, 0.25 ), (torso, 1.75), (upper limb, 1.5) and (lower limb, 0.5). If more than one $I_{i}$ is larger than the threshold, the corresponding likelihood can be computed. Assuming uniform prior distribution of the gestures and common cost function, it is well known that choosing the hypothesis with the largest likelihood gives the Bayesian detector. Different queries using the recognition results were obtained, including the pose recognition, combination (of multiple gestures) and inside-vocabulary (the poses are inside the set of gestures).

\section{Multimodal Feedback \\ 3.1. Audio Feedback}

For our proof of concept work for audio feedback, we decided on a simple A-B-A' pattern that could demonstrate the detection of different features. In the first section only poses that were detected had any affect on the sound. Two different granulated sound sources where mixed through and FFT based spectral attenuation. Each recognized gesture revealed a specific frequency range of the second sound source while at the same time decreasing the spectral energy in the first sound source thereby producing an unmasking of the second source.

Section B of the piece was more improvisatory with short punctuations created through a return of one of the 21 gestures. The sound was produced by various additive synthesis methods with pitch material being selected by a simple genetic algorithm. As time increased between a return of a recognized gesture the GA evolved through a fitness function that selected for increasing dissonance based on Parncutt's roughness model [4]. Also, as the time interval since a previous recognized gesture increased an expansion in movement of sound sources also resulted though changing the avoidance radius of a simple flocking algorithm that was mapped to the 5.1 surround setup.

The return of section A was signaled by recognition that the dancer had returned to predominantly using the recognized movement vocabulary. The audio functioned in much the same manner as in the first section A except that this time the frequency ranges of the first sound source was unmasked by each recognized gesture.

\subsection{D Graphical Feedback}

The visual feedback system plays animations from previously recorded motion captures, driven by the recognition system. Movements of a child were captured and saved into files. The choreographer viewed the captures and selected the desired sequences. Each chosen sequence was then associated with the dancer's gestures. Multiple animation sequences were associated with each gesture. The choreographer also characterized each of the dancer's gestures for the purpose of associating different color schemes for the animations triggered by the gesture.

Instead of rendering the animation with a standard wire-frame or shaded style, a softer, less distinct appearance was created for the character. The segments of the body are rendered using translucent, texture- 
mapped polygons that are aligned to face the camera. The number of polygons used depends on the length of the particular segment, in order to create a smooth effect. The texture maps used are painted so that when combined they create a soft, smoky effect.

At runtime the visual system loads all of the selected motion capture files into memory for playback. The program listens to the recognition system via a multicast network protocol. The recognition data are used to trigger the playback of animation. For example, when a gesture is recognized one of the associated animations is chosen for playback. The playback speed, position and color of the animation is varied each time for visual interest. The system allows multiple animations to be displayed, creating interesting interactions between the different sequences. When a surprise event occurs, the system causes the animations that are currently running to be pushed away from the center of the stage. When the dancer stays out of the gesture vocabulary for longer times, a different set of animations are triggered that are larger and less distinct than the gesture animations.

\section{Experimental Results}

The gesture recognition engine is implemented using Visual $\mathrm{C}++$ in the .net environment on a Pentium 4 PC with $2.4 \mathrm{GHz}$ CPU. The motion capture system we use is an eight-camera VICON system with frame rate of $120 \mathrm{~Hz}$. In our experiments, we have used a set of gestures named 21, created by a world renowned choreographer Bill $\mathrm{T}$. Jones. This set of gestures has 21 different gestures, each with specific meaning. In our experiment, two dancers performed 4 trials each. Each trial contains a full run of the 21 gestures. From each dancer, 3 trails were used for training and the remaining one for testing. Accurate recognition results have been achieved. The false alarm is less than one percentage. Table 2 gives the associated recognition rates, which is the ratio of correctly recognized frame number and the total frame number. Table 2: Gesture Recognition Rate (\%)

\begin{tabular}{|c|c|c|c|c|}
\hline Trials & 1 & 2 & 3 & 4 \\
\hline Dancer 1 & 94.7 & 100 & 100 & 100 \\
\hline Dancer 2 & 100 & 100 & 100 & 99.7 \\
\hline
\end{tabular}

The audio part of the interactive feedback system was implemented using the Max/MSP graphical programming environment extended through the use of several custom made plug-in for reception of the gesture recognition stream, sound granulation, algorithmic spatialization, and control of perceptual roughness/dissonance. Playback was through a multi-channel audio card and 5.1 surround speaker system.

The 3D graphical feedback system achieves good real time performance, with frame rates varying widely, depending on how many animations are playing at once, and how much screen space is covered. With several of the gesture animations playing, the frame rate exceeds 120 frames per second. When several of the large out of vocabulary animations fill the entire screen, the frame rate can drop to $30 \mathrm{fps}$. Typical frame rates during the performance are 70-80 fps. These timings are on a dual processor, 2GHz Apple PowerMac G5, ATI 9800 Pro graphics card. The display screen running at $1024 \times 768$, double buffered, with multi-sample anti-aliasing turned on. The visual feedback system was written in $\mathrm{C}$ using OpenGL, in the Xcode development environment, running on an Apple PowerMac G5. In Figure 7 the image on left shows marker positions and the image on right is texture mapped figure for performance display.

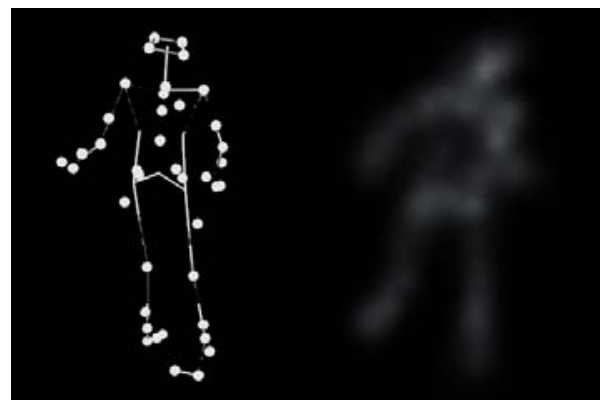

Figure 7: A gesture (left) and the related texture mapped figure for performance display

The reported interactive dance system has been successfully demonstrated at the second annual meeting of NATN in November 2003. A video recording of the gesture recognition and the complete demo with multimodal feedback is recorded at http://www.public.asu.edu/ gqian.

\section{Conclusion}

An end-to-end real time multimodal interactive system for dance has been reported in this paper. This system is driven by a gesture recognition engine, which handles missing marker problem and provides real-time recognition results of gestures. The reported system is easy to implement and robust to marker occlusion. Satisfactory interactive dance performances have been created and presented using this system.

\section{References}

[1] Camurri, A., Hashimoto, S., Ricchetti, M., Ricci, A., Suzuki, K., Trocca, R. and Volpe, G., "EyesWeb: Toward Gesture and Affect Recognition in Interactive Dance and Music Systems”. Computer Music Journal. 24(1), 57-69, 2000

[2] Moore, C.-L., Yamamoto, K. Beyond Words: Movement Observation and Analysis. Gordon and Breach Science Publishers, New York, 1988.

[3] Woo, W., Kim, N., Wong, K., and Tadenuma M. "Sketch on Dynamic Gesture Tracking and Analysis Exploiting Vision-based 3D Interface”. Proc. SPIE PW-EI-VCIP'01, vol. 4310, pp. 656-666, 2000

[4] Parncutt, R. Harmony: A Psychoacoustical Approach. Berlin: Springer-Verlag. 1989 
